\title{
Locating attractiveness in the face space: Faces are more attractive when closer to their group prototype
}

\author{
TIMOThy PotTer \\ Université Catholique de Louvain, Louvain, Belgium \\ and Fonds de la Recherche Scientifique, Brussels, Belgium \\ AND \\ Olivier Corneille \\ Université Catholique de Louvain, Louvain, Belgium
}

\begin{abstract}
Face attractiveness relates positively to the mathematical averageness of a face, but how close attractive faces of varying groups are to their own and to other-group prototypes in the face space remains unclear. In two studies, we modeled the locations of attractive and unattractive Caucasian, Asian, and African faces in participants' face space using multidimensional scaling analysis. In all three sets of faces, facial attractiveness significantly increased with the absolute proximity of a face to its group prototype. In the case of Caucasian and African faces (Study 1), facial attractiveness also tended to increase with the absolute proximity of a face to the other-group prototype. However, this association was at best marginal, and it became clearly nonsignificant when distance to the own-group prototype was controlled for. Thus, the present research provides original evidence that average features of faces contribute to increasing their attractiveness, but only when these features are average to the group to which a face belongs. The present research also offers further support to face space models of people's mental representations of faces.
\end{abstract}

Previous research on faces has shown a relation between facial attractiveness and the mathematical averageness of a face (e.g., Langlois \& Roggman, 1990). Rhodes, Harwood, Yoshikawa, Nishitani, and McLean (2002) also showed that people from different social groups (e.g., Caucasian and Asian) show high intergroup correlations in attractiveness ratings of the same mathematically average faces, regardless of the group memberships of the raters or of the faces being rated. Yet, relatively little is known about how people's mental representations of faces relate to the faces' attractiveness. In particular, no research to date has examined how faces that vary in attractiveness are represented relative to mathematical averages (i.e., to prototypes) for various ethnic groups, a gap that the present research was designed to address.

One popular framework used to understand how faces are perceived, encoded, and retrieved is the face space (Lewis, 2004; Valentine, 1991). This space is akin to a mental "map" in which each dimension is normally distributed and holds information necessary to remembering and recognizing a face. Busey (1998) showed that the face space can be modeled adequately using multidimensional scaling (MDS) analysis. He used faces of bald men and their morphs in a similarity judgment task involving all possible combinations of faces. The morphs appeared more typical than their parent faces and were found closer to the center, in line with predictions made by the face space model. The morphs were also found to be less typical than predicted, consistent with recent research on attractor field models that has shown greater perception of dissimilarity between two faces in more populated, highdensity zones (Corneille, Hugenberg, \& Potter, 2007; Tanaka \& Corneille, 2007; Tanaka, Giles, Kremen, \& Simon, 1998).

Concerning race in the face space, Byatt and Rhodes (2004) showed using MDS that, for Caucasian participants, Asian faces cluster in the face space more than Caucasian faces, and this clustering explained why Asian faces were less well recognized. As for attractive faces in the face space, Potter, Corneille, Ruys, and Rhodes (2007), using real faces, recently showed that, because of their perceived similarity, attractive faces cluster in the face space more than unattractive faces, regardless of group. This clustering makes them lie in higher-density zones that require more competition for activation, which accounts for their decreased recognition in comparison with unattractive faces (Lewis, 2004; see also Light, Hollander, \& Kayra-Stuart, 1981). However, Potter et al.'s study had a heterogeneous, non-Caucasian group of faces (involving Asians, Africans, and North Africans) and did not include prototypes from

T. Potter, timothy.potter@uclouvain.be 
the various groups, so it was not possible for the authors to determine whether attractive faces were closer to the average face of an attractive face's own specific group. Thus, it remains to be known where faces that vary in attractiveness and group would be located in the face space relative to own- and other-group prototypes.

In the present research, we used software from Singular Inversions called FaceGen 3.1 (www.facegen.com) that has recently been used in research on face perception (Russell, Sinha, Biederman, \& Nederhouser, 2006; SchulteRüther, Markowitsch, Fink, \& Piefke, 2007; Shimojo, Simion, Shimojo, \& Scheier, 2003). This has enabled us to generate materials suitable to answering our questions. In two studies, we implemented a similarity judgment task involving the most attractive and unattractive faces of two groups, as well as their respective prototypes. We ran an MDS analysis in order to investigate our research questions. Below, we introduce the main questions we were interested in for the present research. Next, we explain why we chose to address these questions using computergenerated faces. Finally, we report and discuss the two studies designed to fit our present research interests.

\section{The Present Research Questions}

1. Are attractive faces closer to their group prototypes than are unattractive faces (Hypothesis 1A), and do attractiveness ratings of faces increase with proximity to the own-group prototype, irrespective of distance to the other-group prototype (Hypothesis 1B)?

If supported, these hypotheses would extend previous research that has shown that category prototypes are cognitively pleasing (e.g., Halberstadt \& Rhodes, 2000). Indeed, being a prototypical, "good" exemplar of its specific group (e.g., of cars or clocks - and perhaps, in the present research, of an ethnic group) makes a face easier to process and more attractive (Winkielman, Halberstadt, Fazendeiro, \& Catty, 2006).

2. Are attractive faces closer to the other-group prototype than are unattractive faces (Hypothesis 2A), and do attractiveness ratings of faces increase with proximity to the other-group prototype, irrespective of distance to the own-group prototype (Hypothesis $2 \mathrm{~B})$ ?

If supported, these hypotheses would suggest that facial features that transcend group differences play a role in attractiveness. This would be consistent with previous research that has shown that morphs between faces of different races (Asian and Caucasian) are judged as more attractive than the parent faces used to create them (Rhodes et al., 2005). For example, the most attractive Caucasian faces could lie closer not only to the Caucasian, but also to the African, prototype. The corollary is that, for several faces that are equidistant to their group prototype, the most attractive of the faces would be the ones closest to the other-group prototype.

\section{Why Did We Use Computer-Generated Faces?}

First, in order to model a face space, we used MDS analysis and implemented a similarity judgment task between all possible pairs of the faces to be located. When making similarity judgments, it is desirable to use standardized faces, in order to make sure that participants focus on $f a-$ cial features and not on some random salient feature also present in a picture. Using 3-D faces allowed us to keep them bald (see also Busey, 1998) without having to use an oval "cut-in" to crop the pictures and also to keep other features perfectly standardized (e.g., facial orientation, lighting conditions, and skin texture). The disadvantage was that this procedure prevented us from having high absolute attractiveness ratings, because bald faces have a tendency to be rated as less attractive (Cash, 1999).

Second, we wanted to implement prototypes. A prototype derived from morphing tends to be blurred, which can create problems when attempting to localize it in a face space (Byatt $\&$ Rhodes, 2004). The current version of the FaceGen software is sufficiently powerful to extract a mathematically average face for specific groups that is just as standardized as the other faces.

Admittedly, using artificial rather than real faces does create some disadvantages. However, we believe there is no theoretical reason why people who rate the attractiveness of artificial faces would not use the same criteria they use to rate real faces. Conclusions derived from using artificial stimuli remain psychologically meaningful. For a similarity judgment task, we believe that the advantages of using artificial faces ultimately outweigh the disadvantages.

\section{The Present Studies}

In Study 1, we located in face space Caucasian and African faces that varied in attractiveness. Study 2 replicated and extended Study 1, this time using Caucasian and Asian faces. Because these studies were highly similar in terms of the designs and analyses, we introduce and analyze them together in the following sections.

\section{METHOD}

\section{Participants}

Eighteen undergraduate Caucasian psychology students (in Study 1, 16 female, 2 male; in Study 2, 17 female, 1 male) participated in collective computer sessions in exchange for course credit. A large sample size is not necessary when conducting an MDS analysis (see also Byatt \& Rhodes, 2004) in order to produce coherent and meaningful data. We did not find it necessary to include equal numbers of male and female students, considering the high intergender agreement in attractiveness ratings reported in the literature and in similar past work using the MDS technique (Rhodes \& Zebrowitz, 2002; see also Byatt \& Rhodes, 2004).

\section{Materials}

We used the FaceGen 3.1 software, which is based on statistical modeling of a sample of 300 real faces varying in gender and ethnicity, using a process similar to the one used by Blanz and Vetter (1999). The software uses more than 100 normally distributed dimensions to model facial features (i.e., eye size, cheekbones, mouth, lips, etc.). Faces may be generated randomly. In order to generate faces from a specific ethnic group, dimensions that are key to that group may be kept constant with a feature called rand lock. The program can compute for each ethnic group a face that is mathematically average in terms of all of the dimensions used. These prototypes are computed by applying a nonrigid transform to each face to put it in correspondence with a base face. We did not use the detail textures feature, in order to keep skin textures identical. 

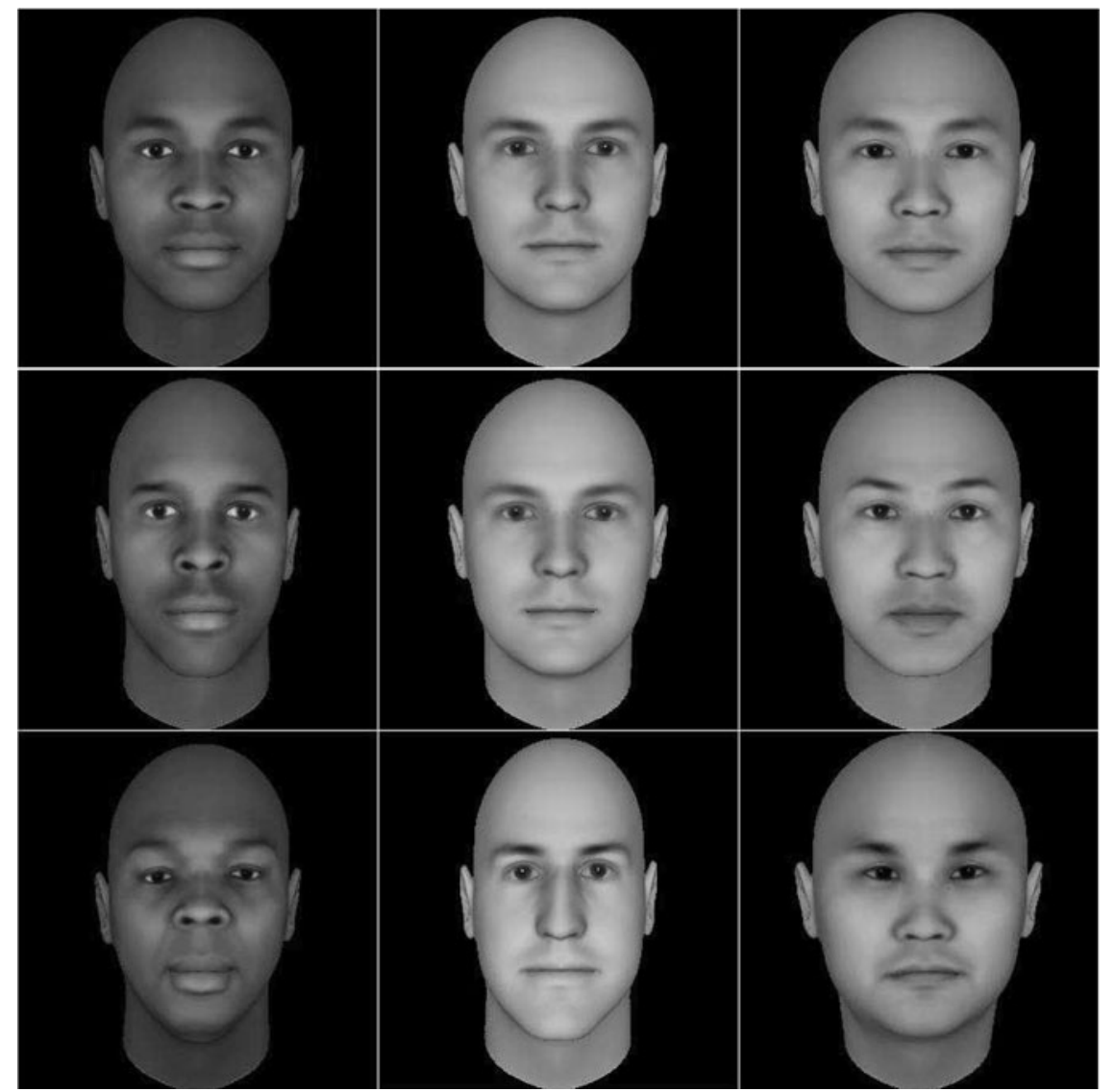

Figure 1. Sample of the materials used. From top to bottom are the three prototypes, representing a sample of attractive and unattractive faces. From left to right are African, Caucasian, and Asian faces.

We randomly generated 60 male faces for three ethnic groups (Caucasian, African, and Asian), resulting in 180 faces. We pretested these faces for attractiveness (on a scale from 1 to 7 ) on 40 Caucasian college students. Interrater agreement was high (Caucasian $\alpha=$ .89 , African $\alpha=.85$, Asian $\alpha=.89$ ). We then selected the 10 most attractive and 10 most unattractive faces per group according to the mean ratings, with means and variances equalized across groups $[M=3.7$ for both sets of attractive faces and $M=2.2$ for both sets of unattractive faces, with $S D=0.4$ for all sets; the face sets in each group differed significantly in attractiveness, with all $t(18) \mathrm{s}>8$ and all $p \mathrm{~s}<.001]$. Finally, we extracted each group's mathematical average (i.e., the prototype; see Figure 1). In Study 1, we used Caucasian and African faces, and in Study 2, we used Caucasian and Asian faces. We effectively used 42 faces for each study (10 attractive Caucasian, 10 unattractive Caucasian, 1 Caucasian prototype; 10 attractive other-group, 10 unattractive other-group, and 1 othergroup prototype).

\section{Procedure}

We used a similarity judgment task to generate data suitable for modeling a face space using MDS. The participants were seated in front of a PC-compatible computer, and all instructions were presented on one screen. The participants were told that they would make similarity judgments between pairs of faces on a Likert-type scale from 1 (extremely dissimilar) to 7 (extremely similar) and that they would have breaks every 100 presentations (the 42 faces yielded 861 pairs). We asked participants to make spontaneous judgments of similarity on the basis of the faces' traits. Participants were warned that all faces were bald and to focus on the actual facial features.

\section{RESULTS}

We converted similarity ratings to dissimilarity ratings suitable for processing by an MDS PROXSCAL analysis ${ }^{1}$ and ran individual-difference scaling (a form of weighted MDS that accounts for individual differences in the importance attributed to each dimension). This scaling procedure results in a better group fit than simply averaging classic MDS analyses across several participants (see Carroll \& Chang, 1970; Green \& Rao, 1971; Martens \& Zacharov, 2000). A two-dimensional solution was computed in order to graphically represent the face space (see Figure 2A for Study 1 and Figure 2B for Study 2; however, interpretations based on these face spaces should be made with caution, because of their high stress values - in Study 1 , stress $=.28$, and in Study 2, stress $=.30$ ).

The number of dimensions necessary to produce a highly satisfactory fit, and above which any subsequent increase of dimensions would provide only a meager decrease in stress value, was 12 for both studies (Study 1, stress $=.09$; Study 2, stress = .1). We then extracted the coordinates of each face. Using these data, we were able 


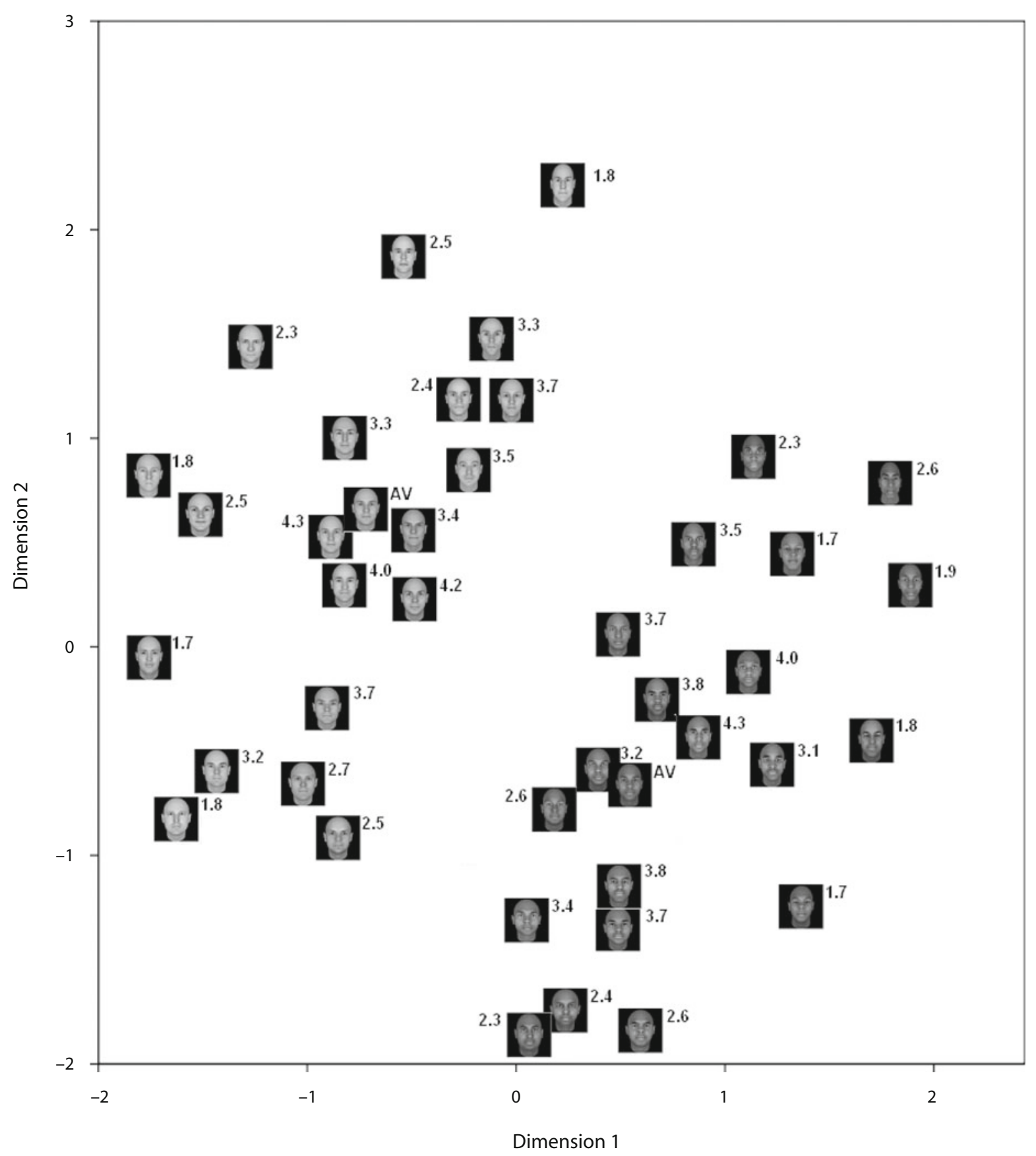

Figure 2A. Two-dimensional MDS representation of the psychological face space in Study 1. Caucasian faces are on the left side and African faces on the right side of the figure. Adjacent to each face lies its average attractiveness rating. "AV" represents the average, prototypical face of the given group. This figure is provided for illustration purposes only and does not represent the final 12-dimensional MDS solution. 


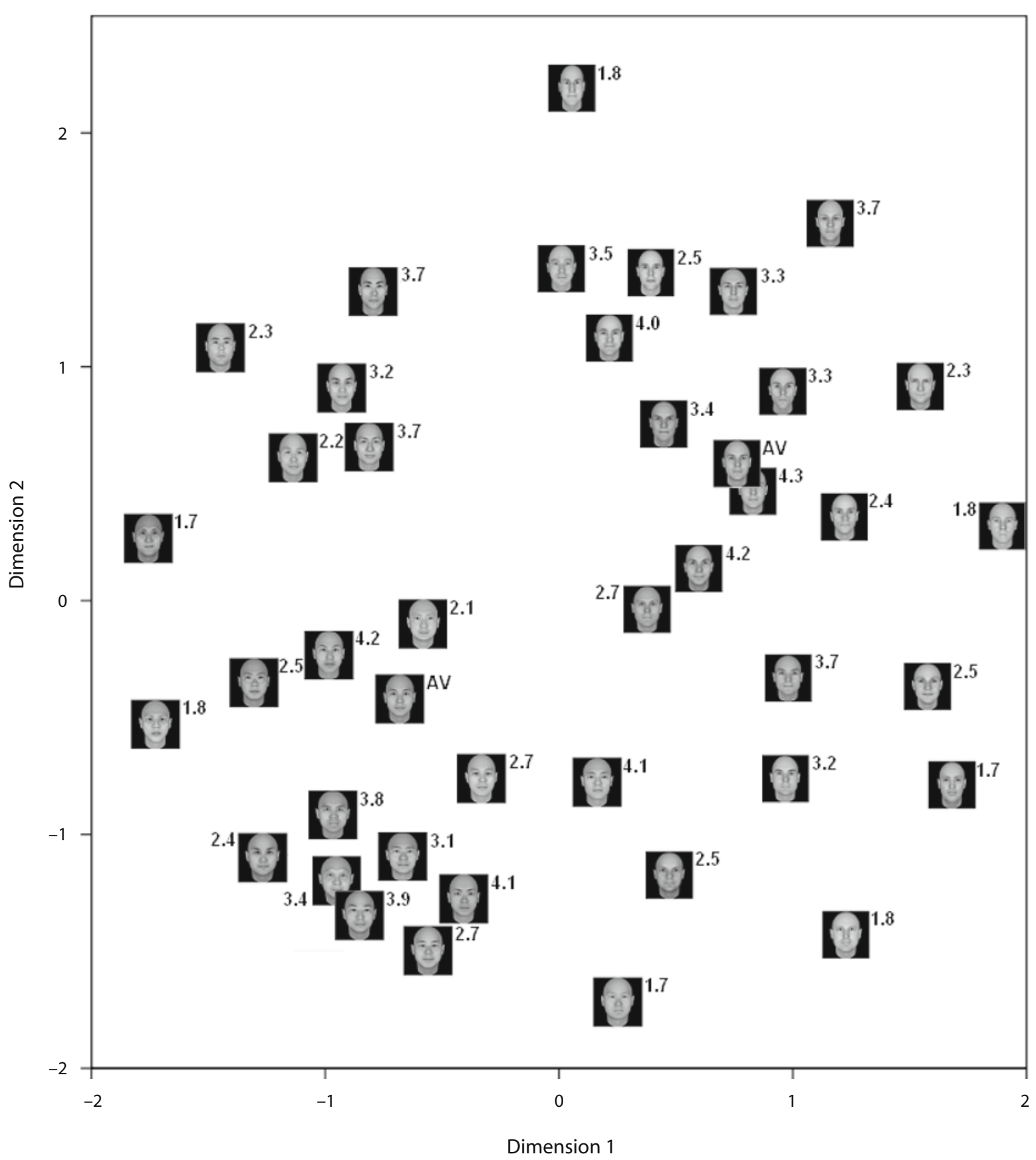

Figure 2B. Two-dimensional MDS representation of the psychological face space in Study 2. Asian faces are on the left side and Caucasian faces on the right side of the figure. Adjacent to each face lies its average attractiveness rating. "AV" represents the average, prototypical face of the given group. This figure is provided for illustration purposes only and does not represent the final 12-dimensional MDS solution. 


\section{A}

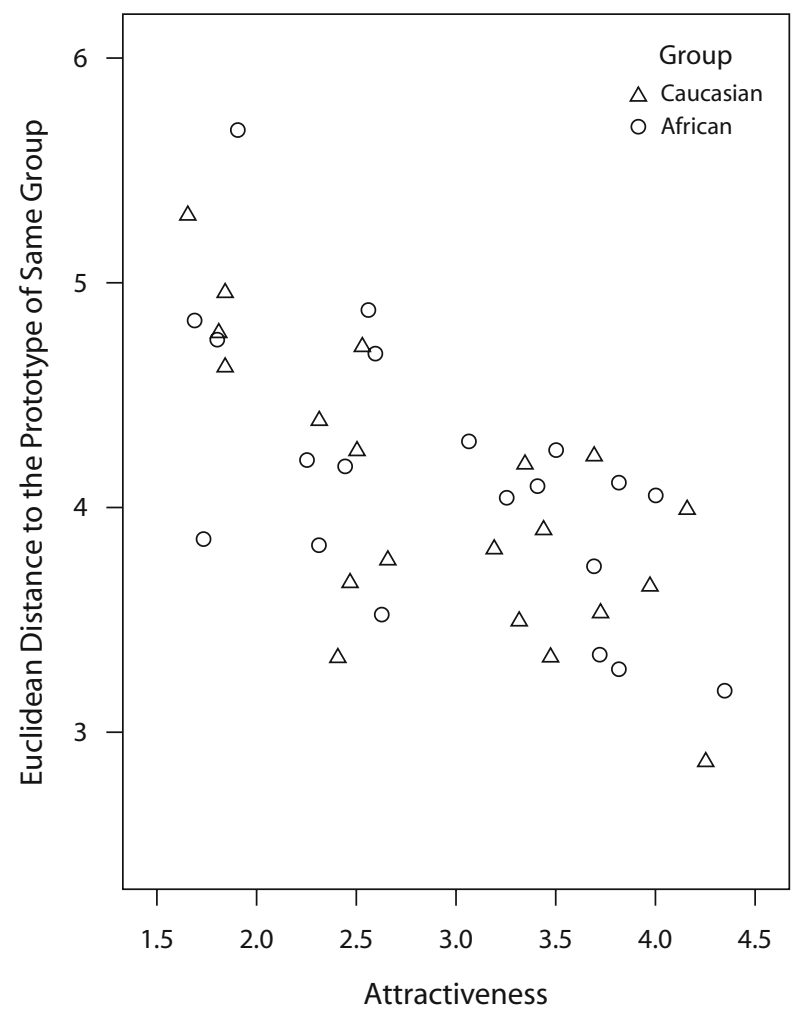

B

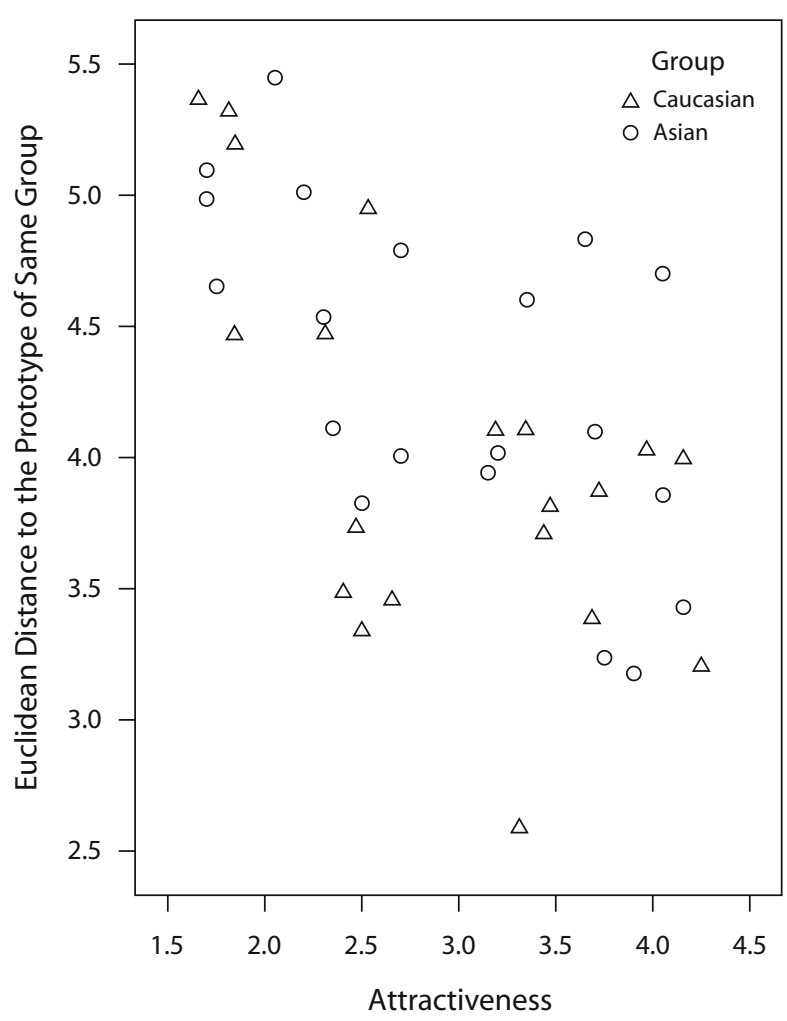

Figure 3. Attractiveness against Euclidean distance to own-group prototype in (A) Study 1 and (B) Study 2.

to compute the Euclidean distance between each face and the respective prototypes. Specifically, we computed the square root of the sum of squared distances between each face and each prototype.

\section{Hypothesis 1A: Attractive Faces Are Closer to Their Group Prototype \\ Than Are Unattractive Faces}

We entered the Euclidean distance between each face and its own-group prototype in a $t$ test with attractiveness as an independent factor. We found that, for both studies, attractive faces of both groups were located closer to the respective prototype than were unattractive faces of the same group [in Study 1, African $t(18)=2.48$, $p<.023$; Caucasian $t(18)=2.85, p<.011$; in Study 2, Asian $t(18)=2.62, p<.027$; Caucasian $t(18)=2.32$, $p<.032]$.

\section{Hypothesis 1B: Attractiveness Ratings of Faces Increase With Proximity to the Own-Group Prototype, Irrespective of Distance to the Other-Group Prototype}

Attractiveness ratings collected in the pretest were correlated with distance to the prototype of a face's own group, for both groups in both studies (for Study 1, African $r=$ $-.62, p<.003$; Caucasian $r=-.71, p<.001-$ see Figure 3A; for Study 2, Asian $r=-.63, p<.003$; Caucasian $r=-.61, p<.004$ - see Figure 3B). Distance to the owngroup prototype and distance to the other-group prototype were correlated in Study 1 for African faces $(r=.58, p<$ $.007)$ and were marginally so for Caucasian faces $(r=.42$, $p<.064)$. In Study 2, the distances were not correlated for Asian faces $(r=.053, p<.825)$ but were for Caucasian faces $(r=.66, p<.002)$.

We then computed partial correlations to control for distance to the other-group prototype, which is a more important measure for Hypothesis 1B. The correlations between attractiveness and distance to the own-group prototype remained strong when distance to the other-group prototype was controlled for (in Study 1, African $r=-.51, p<.025$; Caucasian $r=-.66, p<.001$; in Study 2, Asian $r=-.64$, $p<.003$; Caucasian $r=-.61, p<.006$ ).

\section{Hypothesis 2A: Attractive Faces Are Closer to the Other-Group Prototype Than \\ Are Unattractive Faces}

We entered the distance between each face and the other-group prototype in a $t$ test with attractiveness as an independent factor. We found that, for both groups in both studies, attractive faces were not significantly closer to the other-group prototype than were unattractive faces of the same group [in Study 1, both $t(18) \mathrm{s}<1$, n.s.; in Study 2, Asian $t(18)=1.87, p<.078$; Caucasian $t(18)<1$, n.s.]. 


\section{Hypothesis 2B: Attractiveness Ratings of Faces Increase With Proximity to the Other-Group Prototype, Irrespective of Distance to the Own-Group Prototype}

For both groups in both studies, attractiveness was not significantly correlated with distance to the other-group prototype (for Study 1, African $r=-.42, p<.064$; Caucasian $r=-.36, p<.12$; for Study 2, Asian $r=-.11$, $p<.639$; Caucasian $r=-.26, p<.275)$. Note that the factors were apparently marginally correlated in Study 1. However, when distance to the own-group prototype was controlled for, the correlation between attractiveness and distance to the other-group prototype completely disappeared ( $p \mathrm{~s}>.71$ for both groups).

\section{Complementary Analyses}

All faces, attractive or unattractive, were significantly closer to their own-group than to the other-group prototype for both groups in both studies (all $t \mathrm{~s}>4.24$ and all $p \mathrm{~s}<.002$ ), except for unattractive Asian faces in Study 2, for which this tendency was only marginally significant $[t(9)=1.96, p<.081]$. In addition, and in line with the recent findings of Potter et al. (2007), attractive faces of both groups in Study 1 were more clustered in the psychological space than were unattractive faces [African $t(18)=5.65$, $p<.001$; Caucasian $t(18)=7.58, p<.001]$. In Study 2, only the attractive Caucasians were more clustered than the unattractive faces in their group $[t(18)=9.51, p<.001]$. The difference was not significant for Asian faces, but there was a trend in the same direction $[t(18)=1.34, p<.197]$.

\section{DISCUSSION}

The present research provides original evidence that average features of faces do contribute to increasing a face's attractiveness, but only when these features are average within the group to which the face belongs. Indeed, attractive faces are located closer only to their own-group prototype. Thus, it would seem that when judging a face's attractiveness, people rely on the specific standards of the face's group instead of on the standards of other groups, even of a participant's own group. This finding was replicated across the sets of Caucasian, Asian, and African faces. It extends prior research on the link between attractiveness and mathematical averageness (Langlois \& Roggman, 1990), which equates to prototypicality when only one group is being considered. Note that the present data also replicate with novel computer-generated stimuli the finding of Potter et al. (2007) that attractive faces are more clustered than unattractive faces.

The location of attractive faces both closer to the prototype and in higher-density zones has implications for face memory and face space models (Lewis, 2004; Valentine, 1991). Face space models explain that typical faces lead to higher false alarm and lower hit rates (see also Vokey \& Read, 1992) because they are located in higher density zones requiring more competition for activation and lie closer to the center, represented by the overall average face (i.e., the prototype). Attractive faces are more typical than unattractive faces, and past research has shown that attractive Caucasian faces seem more familiar and are harder to remember than unattractive Caucasian faces (e.g., Corneille, Monin, \& Pleyers, 2005; Light et al., 1981). Our results offer empirical support for the face space conceptualization by showing that attractive faces are located closer to the prototype, reflecting their typicality, and in higher-density zones. However, it remains to be tested whether the memory effects we have mentioned generalize to attractive and unattractive faces from groups other than Caucasians. Considering our findings, we predict that they will, so that faces from any group will be associated with lower memory performance when they are located in zones of higher density and are closer to the group prototype.

However, the fact that face attractiveness increases with the proximity of a face to its group prototype does not imply that mixed-group faces should be systematically judged to be less attractive. In Rhodes et al. (2002), for instance, Caucasian and Asian participants judged Eurasian composite faces as more attractive than either Caucasian or Asian prototypes. Yet, if we considered a pool of mixedgroup faces that were categorized as such (e.g., Eurasian faces), we would hypothesize that the more the faces lay specifically closer to the Eurasian (rather than to either the Caucasian or the Asian) prototype, the more they would be judged as attractive. We could also predict that the advantage for mixed-group faces might not emerge when considering mixed-group faces for which no clear prototype has been formed. The idea that faces from different groups are represented as organized around the prototypes for their specific groups has interesting implications for memory. For instance, Corneille, Huart, Becquart, and Brédart (2004) showed that faces whose race is made ambiguous through morphing with a face from another race are misremembered as being closer to their own-race prototype than they actually are. The same effect applies to faces that are made ambiguous as to gender identity, with memory distortions toward gender prototypes (Huart, Corneille, \& Becquart, 2005).

In our studies, only Caucasian participants were considered. To the extent that no own-race bias was found in the present studies, we do not see this as a major problem. Yet, it may be worthwhile to replicate the present set of findings within other populations. More generally, it would be interesting to examine how our findings translate to people who are highly exposed to faces from various groups. One may hypothesize that people with a wide experience of faces from several groups might develop an additional "prototypical" face in the face space representing the average of faces from several groups. Such a person's perception of attractiveness might possibly shift according to this new, more integrative conception of prototypicality. Relevant to this point is the recent work by Rhodes, Jeffery, Watson, Clifford, and Nakayama (2003), who suggested that our experience of faces shapes what we consider prototypical, which in turn influences what we perceive as being attractive. Our results do not enable us to answer these questions, which future research should address. In any case, the conclusions from this research by no means suggest that attractiveness ratings should be independent from transient context effects. 


\section{AUTHOR NOTE}

We thank Gill Rhodes and Florence Stinglhamber for their comments on previous versions of the manuscript. Correspondence concerning this article may be addressed to T. Potter, Université Catholique de Louvain, PSP-PSOR, 10, Place du Cardinal Mercier, B-1348 Louvain-la-Neuve, Belgium (e-mail: timothy.potter@uclouvain.be).

\section{REFERENCES}

Blanz, V., \& Vetter, T. A. (1999). A morphable model for the synthesis of 3D faces. In SIGGRAPH '99 Computer Graphics Proceedings (pp. 187-194). Boston: Addison-Wesley.

BUSEY, T. A. (1998). Physical and psychological representations of faces: Evidence from morphing. Psychological Science, 9, 476-483.

ByATt, G., \& RhodEs, G. (2004). Identification of own-race and otherrace faces: Implications for the representation of race in face space. Psychonomic Bulletin \& Review, 11, 735-741.

Carroll, J. D., \& Chang, J.-J. (1970). Analysis of individual differences in multidimensional scaling via an $n$-way generalization of "Eckart-Young" decomposition. Psychometrika, 35, 283-319.

CASH, T. F. (1999). The psychosocial consequences of androgenetic alopecia: A review of the research literature. British Journal of Dermatol ogy, 141, 398-405.

Corneille, O., Huart, J., Becquart, E., \& Brédart, S. (2004). When memory shifts towards more typical category exemplars: Accentuation effects in the recollection of ethnically ambiguous faces. Journal of Personality \& Social Psychology, 86, 236-250.

Corneille, O., Hugenberg, K., \& Potter, T. (2007). Applying the attractor field model to social cognition: Perceptual discrimination is facilitated, but memory is impaired for faces displaying evaluatively congruent expressions. Journal of Personality \& Social Psychology, 93, 335-352.

Corneille, O., Monin, B., \& Pleyers, G. (2005). Is positivity a cue or a response option? Warm glow vs. evaluative matching in the familiarity for attractive and not-so-attractive faces. Journal of Experimental Social Psychology, 41, 431-437.

GreEn, P. E., \& RAO, V. R. (1971). Multidimensional scaling and individual differences. Journal of Marketing Research, 8, 71-77.

Halberstadt, J., \& Rhodes, G. (2000). The attractiveness of nonface averages: Implications for an evolutionary explanation of the attractiveness of average faces. Psychological Science, 11, 285-289.

Huart, J., Corneille, O., \& Becquart, E. (2005). Face-based categorization, context-based categorization, and distortions in the recollection of gender ambiguous faces. Journal of Experimental Social Psychology, 41, 598-608.

LANGLois, J. H., \& Roggman, L. A. (1990). Attractive faces are only average. Psychological Science, 1, 115-121.

LEwIS, M. B. (2004). Face-space-R: Towards a unified account of face recognition. Visual Cognition, 11, 29-69.

Light, L. L., Hollander, S., \& KaYra-Stuart, F. (1981). Why attractive people are harder to remember. Personality \& Social Psychology Bulletin, 7, 269-276.

Martens, W. L., \& Zacharov, N. (2000, September). Multidimensional perceptual unfolding of spatially processed speech I: Deriving stimulus space using INDSCAL. Paper presented at the 109th International Convention of the Audio Engineering Society, Los Angeles.
Potter, T., Corneille, O., Ruys, K. I., \& Rhodes, G. (2007). “Just another pretty face": A multidimensional scaling approach to face attractiveness and variability. Psychonomic Bulletin \& Review, 14, 368-372.

Rhodes, G., Harwood, K., Yoshikawa, S., Nishitani, M., \& McLean, I. G. (2002). The attractiveness of average facial configurations: Cross-cultural evidence and the biology of beauty. In G. Rhodes \& L. A. Zebrowitz (Eds.), Facial attractiveness: Evolutionary, cognitive, and social perspectives (pp. 35-58). Westport, CT: Ablex.

Rhodes, G., Jeffery, L., Watson, T. L., Clifford, C. W. G., \& NaKAYAMA, K. (2003). Fitting the mind to the world: Face adaptation and attractiveness aftereffects. Psychological Science, 14, 558-566.

Rhodes, G., Lee, K., Palermo, R., Weiss, M., Yoshikawa, S., Clissa, P., ET AL. (2005). Attractiveness of own-race, other-race, and mixed-race faces. Perception, 34, 319-340.

Rhodes, G., \& Zebrowitz, L. A. (2002). Facial attractiveness: Evolutionary, cognitive, and social perspectives. Westport, CT: Ablex.

Russell, R., Sinha, P., Biederman, I., \& Nederhouser, M. (2006). Is pigmentation important for face recognition? Evidence from contrast negation. Perception, 35, 749-759.

Schulte-RÜther, M., Markowitsch, H. J., Fink, G. R., \& Piefke, M. (2007). Mirror neuron and theory of mind mechanisms involved in face-to-face interactions: A functional magnetic resonance imaging approach to empathy. Journal of Cognitive Neuroscience, 19, 1354-1372.

Shimojo, S., Simion, C., Shimojo, E., \& Scheier, C. (2003). Gaze bias both reflects and influences preference. Nature Neuroscience, 6, 1317-1322.

TanaKa, J. W., \& Corneille, O. (2007). Typicality effects in face and object perception: Further evidence for the attractor field model. Perception \& Psychophysics, 69, 619-627.

Tanaka, J. [W.], Giles, M., Kremen, S., \& Simon, V. (1998). Mapping attractor fields in face space: The atypicality of bias in face recognition. Cognition, 68, 199-220.

VAlentine, T. (1991). A unified account of the effects of distinctiveness, inversion, and race in face recognition. Quarterly Journal of Experimental Psychology, 43A, 161-204.

VoKeY, J. R., \& READ, J. D. (1992). Familiarity, memorability, and the effect of typicality on the recognition of faces. Memory \& Cognition, 20, 291-302.

Winkielman, P., Halberstadt, J., Fazendeiro, T., \& Catty, S. (2006). Prototypes are attractive because they are easy on the mind. Psychological Science, 17, 799-806.

\section{NOTE}

1. We originally used an ALSCAL procedure with 6 outputted dimensions. However, stress values were not satisfactory with this dimensionality. Unfortunately, ALSCAL is limited to 6 dimensions in SPSS software. Turning to a PROXSCAL procedure, also included in the SPSS software, allowed us to increase the number of dimensions to 12 , and this gave us better stress while also allowing a number of dimensions closer to those in previous reports, suggesting that the dimensionality of the face space is between 15 and 22 (Lewis, 2004).

(Manuscript received June 7, 2007; revision accepted for publication November 28, 2007.) 